\title{
DESENVOLVIMENTO DE PASTAGENS EM INTEGRAÇÃO LAVOURA-PECUÁRIA NA REGIÃO DE GUARAPUAVA-PR
}

\author{
Pasture development in the crop-cattle raising integration in Guarapuava-PR
}

\author{
Denise Bruginski de Carvalho', Márcia Bello², Aníbal de Moraes ${ }^{3}$, \\ Adelino Pelissari ${ }^{4}$, Amadeu Bona Filho ${ }^{5}$

\footnotetext{
${ }^{1}$ Engenheira Agrônoma, Dra., Autônoma. São José dos Pinhais, PR - Brasil. e-mail: denise.bcarvalho@terra.com.br

${ }^{2}$ Engenheira Agrônoma, M. Sc. (in memoriam)

${ }^{3}$ Engenheiro Agrônomo, Dr. Prof. Departamento de Fitotecnia e Fitossanitarismo, UFPR. Curitiba, PR - Brasil. e-mail: anibalm@ufpr.br

${ }^{4}$ Engenheiro Agrônomo, Dr. Prof. Departamento de Fitotecnia e Fitossanitarismo, UFPR. Curitiba, PR - Brasil. e-mail: linopeli@hotmail.com

${ }^{5}$ Médico Veterinário, Dr. Prof. Departamento de Zootecnia. UFPR. Curitiba, PR - Brasil. e-mail: bona@ufpr.br
}

\begin{abstract}
Resumo
Este trabalho foi desenvolvido nos princípios de enfoque sistêmico em propriedades que utilizam o sistema de integração lavoura-pecuária na região de Guarapuava-PR, Brasil. O objetivo foi verificar a produção de massa seca de pastagens de inverno, o resíduo médio final que é utilizado como cobertura morta para o cultivo de lavoura de verão e o desenvolvimento de pastagens em áreas mantidas somente com pasto. Os dados foram coletados no período entre dezembro/2000 a outubro/2001, registrando-se a temperatura e precipitação pluvial. Apesar do baixo resíduo de massa seca das pastagens de inverno no mês de junho, houve predominância de manutenção de massa seca sempre acima de $2000 \mathrm{~kg} \mathrm{ha}^{-1}$. Nas áreas com apenas pastagens, a melhor taxa de acúmulo de massa seca no período foi da área previamente cultivada com sorgo, acumulando um total de $16.996 \mathrm{~kg} \mathrm{ha}^{-1}$. As áreas cultivadas com mistura de espécies de inverno apresentaram maior acúmulo total de massa seca. O contraste nas condições ambientais entre propriedades relacionado ao manejo e características de solo, distintos em cada área estudada, está associado ao crescimento diferenciado de pastagens.
\end{abstract}

Palavras-chave: Clima; Enfoque sistêmico; Massa seca; Sorgo. 


\begin{abstract}
This work was accomplished in systemic focus principle in properties located in Guarapuava$P R$, Brazil in area under the crop-cattle raising integration system. The objective was to check the dry matter production of the winter pasture, the average residue of mulch for summer cropping and the plant development in the areas only maintained with pasture. The data was collected in the period from December/2000 to October/2001 and the precipitation and temperature were registered. In spite of the low dry matter residue in the month of June, there was a predominance of maintenance of dry matter above $2000 \mathrm{~kg} \mathrm{ha}^{-1}$. In the areas kept only with pasture, the best rate of dry matter accumulation in the period was in the area previously cultivated with sorghum, accumulating a total of $16,996 \mathrm{~kg} \mathrm{ha}$. The areas cultivated with mixed winter species resulted in higher amount of dry matter accumulation. The contrast considering the environmental conditions among properties related to the handling and soil characteristics was associated to different pastures growth.
\end{abstract}

Keywords: Climate; Dry matter; Systemic focus; Sorghum.

\title{
INTRODUÇÃO
}

A busca por sistemas sustentáveis na atividade agropecuária aponta para a diversidade dentro da propriedade rural, onde o sistema de integração lavoura-pecuária pode ser considerado como boa opção. A interação existente entre os resíduos utilizados para alimentação animal e os dejetos animais aproveitados para nutrição vegetal fazem com que a energia empreendida retorne ao sistema.

O plantio de pastagem anual durante a safra de inverno no Paraná mostra-se como uma interessante alternativa econômica para substituir o trigo e o milho safrinha, por apresentar menor risco de produção e menor desembolso por hectare (CANZIANI; GUIMARÃES, 2007).

Este trabalho faz parte de um projeto multidisciplinar, desenvolvido nos princípios do enfoque sistêmico, que busca esclarecer os motivos da lenta expansão do sistema de integração lavourapecuária na região de Guarapuava no estado do Paraná. O enfoque sistêmico permite uma análise criteriosa dos entraves à adoção desta tecnologia nos sistemas de produção e uma maior interação entre todos os agentes envolvidos: agropecuaristas, pesquisadores, extensionistas e dirigentes de cooperativas (CARVALHO; BELLO, 2004).

O sistema de integração lavoura-pecuária preconiza a utilização da pastagem de inverno para pastejo e também para formação de cobertura para instalação do cultivo de verão. A formação de pastagens hibernais de azevém e aveia torna viável a terminação de bovinos durante a entressafra e surge como alternativa para melhorar os índices zootécnicos da atividade pecuária na região subtropical da América do Sul, sem comprometer a necessidade de palha para o sistema de plantio direto, observados os ajustes de lotação (MORAES et al., 2007).

A necessidade de uma boa quantidade de palhada para manutenção dos princípios de utilização do sistema de plantio direto e a observância da melhor data para semeadura de grãos, obedecendo o zoneamento agroclimático, podem parecer um entrave para utilização do sistema de integração lavoura pecuária, já que para obter-se animais com peso e qualidade desejáveis pelo mercado local existe a necessidade de um período de engorda que extrapola a época em que se deve retirá-los da área destinada à integração lavoura-pecuária. Nesse sentido, há necessidade de melhores conhecimentos acerca do desenvolvimento de pastagens em áreas onde ocorre a integração entre agricultura e pecuária e também em áreas usadas somente com pasto durante todo ano para terminação animal. O monitoramento de dados relacionados com as variações climáticas no local de estudo é de grande importância, já que no enfoque sistêmico de pesquisa busca-se uma extensão dos aspectos abordados para compreensão de um determinado problema. 
A influência do ser humano nos agroecossistemas é muito intensa, havendo grande investimento energético, seja na forma de fertilizantes, combustíveis ou do próprio trabalho. A manipulação das condições climáticas é possível em ambientes controlados, entretanto, os fatores ambientais ainda representam uma barreira limitante para segurança no investimento agropecuário.

A temperatura e disponibilidade hídrica influenciam diretamente vários processos fisiológicos das plantas, que são bastante estudados e entendidos por pesquisadores, principalmente no que diz respeito ao zoneamento agroclimático, mas muitas vezes não-considerados por proprietários rurais que comparam rendimentos obtidos na sua propriedade com outros proprietários da mesma região ou município.

O objetivo do presente trabalho foi verificar a produção de massa seca de pastagens de inverno, o resíduo médio final que é utilizado como cobertura morta para o cultivo de lavoura de verão e o desenvolvimento de pastagens em áreas mantidas somente com pasto na Região de Guarapuava-PR.

\section{MATERIAL E MÉTODOS}

Os dados foram coletados na Região de Guarapuava-PR, em áreas selecionadas dentro de seis fazendas que utilizam o sistema de integração lavoura-pecuária. Foi realizada uma estimativa de resíduo de massa seca, durante o período de desenvolvimento da pastagem de inverno, compreendido entre os meses de junho a outubro de 2001.

As seis fazendas denominadas por letras de $\mathrm{A}$ a $\mathrm{F}$ não foram submetidas à nenhuma interferência de manejo ou do plano de rotação de culturas. No período estudado, as áreas monitoradas das fazendas estavam sendo cultivadas com aveia preta (Avena strigosa Schreb.): propriedade "A"; aveia preta e azevém (Lolium multiflorum Lam.): "B"; aveia preta, azevém e trevo branco (Trifolium repens L.): "C", "D", "E" e "F".

O método do disco foi utilizado, conforme sugerido por Barcellos (1990), com as calibrações feitas após o corte, secagem e pesagem de doze amostras utilizadas para construção de curvas de calibração, por meio de equações de regressão linear. A média das leituras feitas em 50 pontos ao acaso dentro da área delimitada foi usada para o cálculo do resíduo de massa seca por hectare.

Adicionalmente foram selecionadas áreas mantidas com pastagem durante o ano todo, sendo que no período estudado estavam sendo cultivadas com campo nativo melhorado (A); tifton (Cynodon sp.) no verão e aveia preta + azevém no inverno (B); sorgo forrageiro (Sorghum vulgare Pers.) no verão e aveia preta + azevém + trevo branco no inverno (C); tifton o ano todo (D); hemartria (Hemarthria sp.) o ano todo (E); e campo nativo + pensacola (Paspalum notatum Flügge) (F).

Foi realizada estimativa da taxa de acúmulo da pastagem por meio da técnica de triplo emparelhamento de gaiolas, conforme Moraes, Moojen e Maraschin (1990), com utilização de pequenas áreas de exclusão, obtidas por meio do uso de gaiolas de ferro e arame, com dimensões da base de 0,70 $\mathrm{X} 0,70 \mathrm{~m}$ e altura de $1,0 \mathrm{~m}$. Foi utilizada uma gaiola para cada área monitorada. O procedimento para a amostragem consistiu na escolha, ao acaso, de duas áreas semelhantes em forragem, uma recebendo a proteção da gaiola e outra permanecendo acessível ao pastejo. A cada 28 dias, novas áreas eram escolhidas, repetindo-se o processo.

O acúmulo de massa seca foi calculado pela equação geral $=[G x-F(x-1)]$, onde $G x$ representa a massa seca ha ${ }^{-1}$ dentro da gaiola na amostragem $x ; F(x-1)$ é igual a massa seca ha ${ }^{-1}$ fora da gaiola na amostragem $x-1$. O cálculo da taxa de acúmulo da massa seca foi feito por meio da equação:

$$
T x=\frac{G i-F(i-1)}{n}
$$

Onde: $T x=$ taxa de acúmulo diário no período $x ; G i=$ massa seca em $\mathrm{kg}$ ha ${ }^{-1}$ dentro da gaiola no instante $i ; F(i-1)=$ massa seca ha ${ }^{-1}$ fora da gaiola no instante $i-1 ; n=$ número de dias do período (CAMPBELL, 1966). 
Os registros de temperatura máxima, temperatura mínima e precipitação pluvial foram realizados diariamente durante o período compreendido entre dezembro de 2000 e maio de 2001 nas propriedades B e D. Nas demais propriedades, houve muitas falhas no registro dos dados que foram desconsiderados. Os termômetros e pluviômetros foram instalados próximos das áreas monitoradas que, durante o período estudado, estavam ocupadas com tifton.

A saturação por bases $(\mathrm{V} \%)$ na camada de 0 a $10 \mathrm{~cm}$ do solo da área monitorada da fazenda B em dezembro de 2000 foi de $57 \%$ e da fazenda D foi de $51 \%$. A textura do solo nas duas áreas estudadas é argilosa (CARVALHO et al., 2005).

Foram realizadas médias simples entre a temperatura máxima e a temperatura mínima diária para obtenção da temperatura média diária e somatório da precipitação diária para obtenção da precipitação mensal.

\section{RESULTADOS E DISCUSSÃO}

As avaliações realizadas nas pastagens de inverno mostram que a estimativa de resíduo na primeira época de avaliação apresenta uma situação desfavorável no estabelecimento das pastagens, já que as propriedades $\mathrm{A}$ e $\mathrm{C}$ ainda não tinham um cultivo estabelecido, devido ao atraso na semeadura e nas propriedades D, E e F a estimativa de resíduo foi inferior a $1500 \mathrm{~kg} \mathrm{ha}^{-1}$. conforme dados apresentados na Tabela 1.

TABELA 1 - Estimativa de resíduo de massa seca de pastagens de inverno $\left(\mathrm{kg} \mathrm{ha}^{-1}\right)$ nos meses de junho a outubro, em áreas monitoradas de seis propriedades sob sistema de integração lavoura-pecuária, Região de Guarapuava, 2001

Table 1 - Estimate of winter pasture dry matter residue ( $\left.\mathrm{kg} \mathrm{ha}^{-1}\right)$ from June to October, in monitored areas of six properties under the crop-cattle raising integration system, Guarapuava, 2001

\begin{tabular}{cccccc}
\hline Propriedade & Junho & Julho & Agosto & Setembro & Outubro \\
\hline A & $*$ & 2392 & 3302 & 5032 & 6299 \\
B & 2660 & 2422 & 2069 & 3418 & 7904 \\
C & $*$ & 2004 & 2709 & 2700 & 4722 \\
D & 1487 & 3510 & 3704 & 3748 & $* *$ \\
E & 1318 & 2086 & 2659 & 3614 & 5868 \\
F & 1108 & 4234 & 2824 & 3863 & 7075 \\
\hline
\end{tabular}

* Implantação da pastagem de inverno.

** Implantação antecipada de lavoura de verão.

Nas demais épocas amostradas, o resíduo de massa seca estimado foi sempre superior a $2000 \mathrm{~kg} \mathrm{ha}^{-1}$, o que representa uma condição favorável dentro do manejo de pastagens, considerando a presença de animais nas referidas áreas.

A estimativa realizada no mês de outubro mostra uma boa condição na formação de palhada para o cultivo de verão no sistema de plantio direto, com exceção da propriedade $\mathrm{D}$, que implantou o cultivo de verão ainda no mês de outubro. O resíduo médio estimado no mês de novembro entre as propriedades A, B, C, E e F foi de $6374 \mathrm{~kg} \mathrm{ha}^{-1}$.

Nas áreas com pastagem durante todo ano, observou-se um contraste na produção de massa seca por populações vegetais distintas devido às condições extremamente diversas de ocupação das áreas estudadas, acumulando de $2672 \mathrm{~kg} \mathrm{ha}^{-1}$ na área cultivada com campo nativo (A) até $16.996 \mathrm{~kg} \mathrm{ha}^{-1}$ na área cultivada com sorgo (C), como pode ser observado na Tabela 2. 
TABELA 2 - Taxa de acúmulo de massa seca e acúmulo total de pastagem $\left(\mathrm{kg} \mathrm{ha}^{-1}\right)$ nos meses de dezembro a março (verão/outono), em áreas monitoradas de seis propriedades sob sistema de integração lavoura-pecuária, Região de Guarapuava-PR, 2000/2001

Table 2 - Rate of dry matter accumulation and total pasture accumulation (kg ha ${ }^{-1}$ ) from December to March (summer/ autumn) in monitored areas of six properties under the of crop-cattle raising integration system, Guarapuava, 2001

\begin{tabular}{|c|c|c|c|c|c|c|c|c|c|}
\hline \multirow[t]{2}{*}{ Propriedade } & \multicolumn{2}{|c|}{ Dezembro } & \multicolumn{2}{|c|}{ Janeiro } & \multicolumn{2}{|c|}{ Fevereiro } & \multicolumn{2}{|c|}{ Março } & \multirow{2}{*}{$\begin{array}{l}\text { Acúmulo } \\
\text { total }\end{array}$} \\
\hline & Diário & Mensal & Diário & Mensal & Diário & Mensal & Diário & Mensal & \\
\hline A & 37 & 1048 & 15 & 380 & 29 & 828 & 13 & 416 & 2672 \\
\hline B & 93 & 1020 & 109 & 2824 & 69 & 1928 & 27 & 848 & 6620 \\
\hline $\mathrm{C}$ & 130 & 3644 & 199 & 5176 & 122 & 3420 & 153 & 4756 & 16996 \\
\hline $\mathrm{D}$ & 5 & 136 & 51 & 1336 & 75 & 2108 & 21 & 640 & 4220 \\
\hline $\mathrm{E}$ & 10 & 296 & 59 & 1528 & 78 & 2180 & 21 & 640 & 4644 \\
\hline $\mathrm{F}$ & 83 & 2316 & 32 & 832 & 30 & 836 & 7 & 232 & 4216 \\
\hline
\end{tabular}

Em dezembro foi registrada a menor taxa de acúmulo na área com tifton (D), devido à implantação recente, mas a área com menor acúmulo total durante o período foi a mantida com campo nativo (A), seguida pela área ocupada com campo nativo e pensacola (F).

As condições extremamente diversas de ocupação das áreas estudadas permitem a visualização dos contrastes na produção de massa seca por populações vegetais distintas utilizadas para a mesma finalidade, desconsiderando os tratos culturais, a intensidade de pastejo e o custo de manutenção. Percebe-se que a escolha da espécie associada aos tratos culturais pode determinar melhores condições de pastejo no período, em que há necessidade de terminação dos animais fora das áreas onde ocorre a integração com a agricultura.

No período de outono/inverno houve muitas situações em que os dados não puderam ser coletados. A falta de informação de dados na propriedade $\mathrm{C}$ a partir do mês de maio e na propriedade $\mathrm{B}$ a partir do mês de junho ocorreu devido à retirada das gaiolas para semeadura das espécies de inverno, reduzindo ao mínimo o acúmulo total de massa seca no período (TABELA 3).

TABELA 3 - Taxa de acúmulo de massa seca e acúmulo total de pastagem $\left(\mathrm{kg} \mathrm{ha}^{-1}\right)$ nos meses de abril a junho (outono/inverno), em áreas monitoradas de seis propriedades sob sistema de integração lavoura-pecuária, Região de Guarapuava-PR, 2001

Table 3 - Rate of dry matter accumulation and total pasture accumulation ( $\mathrm{kg} \mathrm{ha}^{-1}$ ) from April to June (autumn/winter) in monitored areas of six properties under the crop-cattle raising integration system, Guarapuava, 2001

\begin{tabular}{cccccccc}
\hline \multirow{2}{*}{ Propriedade } & \multicolumn{2}{c}{ Abril } & \multicolumn{2}{c}{ Maio } & \multicolumn{2}{c}{ Junho } & \multirow{2}{*}{ Acúmulo } \\
& Diário & Mensal & Diário & Mensal & Diário & Mensal & total \\
\hline A & 63 & 2008 & 67 & 1468 & -1 & -1 & 3476 \\
B & 95 & 3052 & 129 & 2836 & -2 & -2 & 5888 \\
C & 20 & 628 & -2 & -2 & -2 & -2 & 628 \\
D & 48 & 1552 & 44 & 972 & -1 & -1 & 2524 \\
E & 18 & 568 & 85 & 1872 & 81 & 2280 & 7160 \\
F & 25 & 812 & -3 & -3 & -3 & -3 & 812 \\
\hline
\end{tabular}

Reforma do talhão.

Implantação de pastagem de inverno.

Venda da propriedade impossibilitou a continuidade da coleta de dados. 
Nas propriedades A e D foram realizados procedimentos para reforma do talhão no mês de junho, o que inviabilizou a continuidade na coleta de dados. A propriedade E, apesar da baixa taxa de acúmulo de massa seca no início do período, foi a que mais acumulou no total. A propriedade F, ocupada com campo nativo e pensacola, foi monitorada até o mês de abril por motivo de venda da propriedade, que apresentava baixa taxa de acúmulo.

Nos meses de abril, maio e junho ainda não havia disponibilidade de pastagem adequada nas áreas de integração lavoura-pecuária (TABELA 1) e percebe-se que nas áreas mantidas somente com pasto apenas as propriedades A e B apresentavam taxa de acúmulo de massa seca acima de $2000 \mathrm{~kg} \mathrm{ha}$ ${ }^{1}$ no mês de abril e na seqüência menor acúmulo mensal. No entanto, esta situação de carência de pastagem neste período não se reflete em problema nas propriedades estudadas pelo fato de trabalharem somente com engorda animal. Os lotes de animais para engorda são adquiridos quando já existe disponibilidade de pasto nas áreas de integração.

As áreas das propriedades A e E passaram por procedimentos de manejo para reforma dos talhões, impossibilitando a coleta de dados nos meses de julho e agosto (TABELA 4).

TABELA 4 - Taxa de acúmulo de massa seca e acúmulo total de pastagem $\left(\mathrm{kg} \mathrm{ha}^{-1}\right)$ nos meses de julho a outubro (inverno/primavera), em áreas monitoradas de seis propriedades sob sistema de integração lavoura-pecuária, Região de Guarapuava-PR, 2001

Table 4 - Rate of dry matter accumulation and total pasture accumulation ( $\mathrm{kg} \mathrm{ha}^{-1}$ ) from July to October (winter/spring) in monitored areas of six properties under the system of crop-cattle raising integration, Guarapuava, 2001

\begin{tabular}{ccccccccccc}
\hline \multirow{2}{*}{ Propriedade } & \multicolumn{2}{c}{ Julho } & \multicolumn{2}{c}{ Agosto } & \multicolumn{2}{c}{ Setembro } & \multicolumn{2}{c}{ Outubro } & \multicolumn{2}{c}{ Acúmulo } \\
& Diário & Mensal & Diário & Mensal & Diário & Mensal & Diário & Mensal & total \\
\hline A & -1 & -1 & 5 & 146 & 23 & 784 & 34 & 840 & 1770 \\
B & $-{ }^{2}$ & -2 & 83 & 2321 & 116 & 3956 & 110 & 2752 & 9032 \\
C & 47 & 1652 & 64 & 1803 & 41 & 1408 & 83 & 2076 & 6939 \\
E & -1 & -1 & -1 & -1 & 12 & 416 & 108 & 2708 & 3124 \\
\hline
\end{tabular}

Reforma do talhão.

2 Implantação da pastagem de inverno.

As propriedades $\mathrm{B}$ e $\mathrm{C}$ apresentaram as maiores taxas de acúmulo total, sendo cultivadas com misturas de espécies de inverno. A presença de trevo branco parece ter favorecido a produção total de massa seca, quando comparado com áreas sem esta mistura, assim como referido por Assmann et al. (2007). O desenvolvimento de pastagens de inverno com maiores taxas de acúmulo é de grande importância nestas áreas mantidas com pasto durante o ano todo, pois podem ser usadas em sistemas de pastejo rotacionado para manutenção de lotação animal adequada nas áreas onde ocorre a integração, evitando o comprometimento da produção de palhada para o sistema de plantio direto, como afirmam Moraes et al. (2007).

A utilização de uma caracterização climática regional que considera homogênea uma região composta muitas vezes de vários municípios pode mascarar importantes variações locais que poderiam interferir diretamente na adoção e continuidade do sistema de integração lavoura-pecuária.

O volume total de chuvas durante o período estudado na propriedade B foi $427 \mathrm{~mm}$, superior à propriedade $\mathrm{D}$, representando $38 \%$ de diferença. Os valores de precipitação pluvial observados nas duas propriedades apresentaram diferenças maiores nos meses de janeiro e fevereiro (FIGURA 1). 


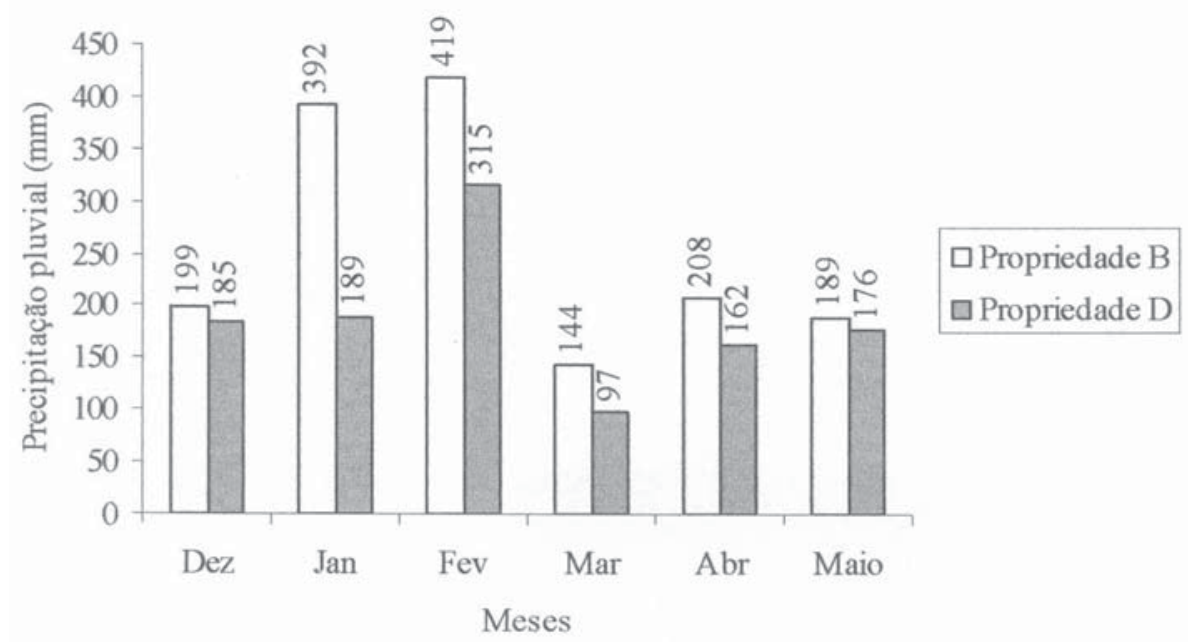

FIGURA 1 - Precipitação pluvial (mm) no período de dezembro de 2000 a agosto de 2001 em áreas monitoradas de duas propriedades sob sistema de integração lavoura-pecuária, Região de Guarapuava, 2000/2001

Figure 1 - Precipitation (mm) in the period from December/2000 to August/2001 in monitored areas of two properties under the crop-cattle raising integration system, Guarapuava, 2000/2001

Os valores referentes à precipitação de forma isolada não podem servir como indicativo preciso da disponibilidade hídrica para as plantas, havendo a necessidade de se considerar valores de evapotranspiração (GLIESSMAN, 2001). As diferenças observadas sugerem que o estudo de um sistema de produção envolvendo integração lavoura-pecuária, além dos fatores relativos ao manejo, deve considerar as interações ambientais não controladas pelos agricultores. As temperaturas médias diárias das duas propriedades foram muito semelhantes, seguindo a tendência normal da época do ano (FUNDAÇÃO INSTITUTO AGRONÔMICO DO PARANÁ, 1984). Os dados podem ser observados na Figura 2.

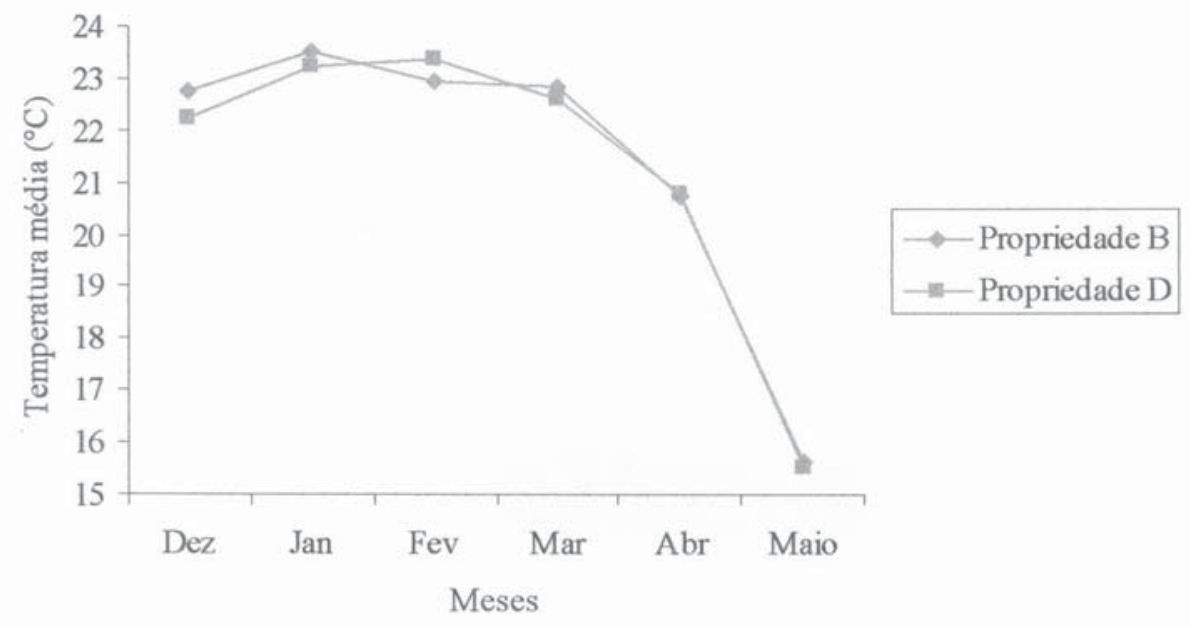

FIGURA 1 - Temperatura média no período de dezembro de 2000 a agosto de 2001 em áreas monitoradas de duas propriedades sob sistema de integração lavoura-pecuária, Região de Guarapuava, 2000/2001

Figure 1 - Average temperature in the period from December/2000 to August/2001 in monitored areas of two properties under the crop-cattle raising integration system, Guarapuava, 2000/2001 
A temperatura mínima registrada durante o período estudado na propriedade $\mathrm{B}$ foi de $8^{\circ} \mathrm{C}$ e na propriedade $\mathrm{D}$ foi de $2^{\circ} \mathrm{C}$. A orientação da encosta e a topografia produzem variações na temperatura, especialmente em nível local, proporcionando microclimas diversos, conforme a orientação da encosta com relação ao sol (GLIESSMAN, 2001). A saturação por bases é considerada por Tomé Júnior (1997) como um indicativo eficiente no nível de fertilidade dos solos. As duas propriedades estudadas apresentaram níveis semelhantes de saturação por bases, acima de $50 \%$, fato que classifica os solos como eutróficos. Embora a fertilidade dos solos influencie diretamente a produção de massa seca de plantas, o manejo adotado e as condições climáticas de uma região podem interferir na expressão do potencial de produção de massa seca.

$\mathrm{O}$ acúmulo total de massa seca da pastagem de tifton desenvolvida na propriedade $\mathrm{B}$, no período de dezembro de 2000 a maio de 2001, foi estimado em $12.508 \mathrm{~kg} \mathrm{ha}^{-1}$. A pastagem da propriedade D acumulou neste mesmo período $6.744 \mathrm{~kg} \mathrm{ha}^{-1}$. Não foram feitos registros de adubações nestes locais durante o período do estudo. Na propriedade D ocorreu menor precipitação pluvial, bem como temperaturas mínimas mais baixas, o que possivelmente contribuiu para a menor produção de massa seca da pastagem, quando comparada com a produção obtida na propriedade B.

Desta forma, o acompanhamento local das variações climáticas, considerando-se as particularidades de cada propriedade, é uma atividade essencial para o melhor entendimento de todos os fatores que, dentro de um enfoque sistêmico, podem interferir na sustentabilidade da produção agropecuária.

\section{CONCLUSÕES}

As pastagens de inverno cultivadas nas áreas de integração lavoura-pecuária monitoradas apresentaram resíduo de massa seca acima de $2000 \mathrm{~kg} \mathrm{ha}^{-1}$. As áreas cultivadas com misturas de espécies de pastagens de inverno apresentaram maior produção de massa seca.

Nas áreas com apenas pastagem monitoradas, a melhor taxa de acúmulo de massa seca registrada no período foi da área cultivada previamente com sorgo, acumulando um total de $16.996 \mathrm{~kg} \mathrm{ha}^{-1}$.

O acompanhamento climático local é uma atividade essencial para o melhor entendimento de todos os fatores que, dentro de um enfoque sistêmico, podem interferir na sustentabilidade da produção agropecuária.

\section{REFERÊNCIAS}

ASMANN, J. M. et al. Acúmulo de matéria seca em sistema de integração lavoura pecuária em presença e ausência de trevo e nitrogênio. In: SIMPÓSIO INTERNACIONAL EM INTEGRAÇÃO LAVOURA-PECUÁRIA, 1., 2007, Curitiba. [Anais eletrônicos...] Curitiba: UFPR/UFRGS/ OHIO STATE, 2007. 1 CD-ROM.

BARCELlOS, A. O. Avaliação de métodos para estimativas de massa de forragem em condições de pastejo. 1990. 181 f. Dissertação (Mestrado em Zootecnia) - Faculdade de Agronomia, Universidade Federal do Rio Grande do Sul, Porto Alegre; 1990.

CAMPBELL, A. G. Grazed pastures parameters. I. Pasture dry-matter production and availability in a stocking rate and grazing management experiment with dairy cows. Journal of Agricultural Science, Cambridge, v. 67, p. 211-216, 1966.

CANZIANI, J. R.; GUIMARÃES, V. D. A. Análise da viabilidade econômica da pecuária de corte no "sistema de integração lavoura-pecuária" em substituição às culturas de trigo e milho safrinha no Estado do Paraná. In: SIMPÓSIO INTERNACIONAL EM INTEGRAÇÃO LAVOURAPECUÁRIA, 1., 2007, Curitiba. [Anais eletrônicos...] Curitiba: UFPR/UFRGS/OHIO STATE, 2007. 1 CD-ROM 
CARVALHO, D. B.; BELLO, M. Enfoque sistêmico como estratégia de estudo para expansão da integração lavoura-pecuária na Região de Guarapuava-PR. Revista Acadêmica: Ciências Agrárias e Ambientais, Curitiba, v. 2, n. 1, p. 31-34, 2004.

CARVALHO, D. B. et al. Fertilidade do solo em integração lavoura-pecuária na Região de Guarapuava/PR. Revista Acadêmica: Ciências Agrárias e Ambientais, Curitiba, v. 3, n. 1, p. 57-65, 2005.

FUNDAÇÃO INSTITUTO AGRONÔMICO DO PARANÁ. Cartas climáticas do Estado do Paraná. Londrina: IAPAR, 1984. 45 p.

GLIESSMAN, S. R. Agroecologia: processos ecológicos em agricultura sustentável. Porto Alegre: Universidade/UFRGS, 2001. 653 p.

MORAES, A. et al. Sistemas de integração lavoura-pecuária no Subtrópico da América do Sul: exemplos do sul do Brasil. In: SIMPÓSIO INTERNACIONAL EM INTEGRAÇÃO LAVOURAPECUÁRIA, 1., 2007, Curitiba. [Anais eletrônicos...] Curitiba: UFPR/UFRGS/OHIO STATE, 2007. 1 CD-ROM.

MORAES, A.; MOOJEN, E. L.; MARASCHIN, G. E. Comparação de métodos de estimativas de taxas de crescimento em uma pastagem submetida a diferentes pressões de pastejo. In: REUNIÃO ANUAL DA SOCIEDADE BRASILEIRA DE ZOOTECNIA, 27., 1990, Campinas. Anais... Piracicaba: FEALQ, 1990. p. 332.

TOMÉ JÚNIOR, J. B. Manual para interpretação de análise de solo. Guaíba: Agropecuária, 1997. 247 p. 